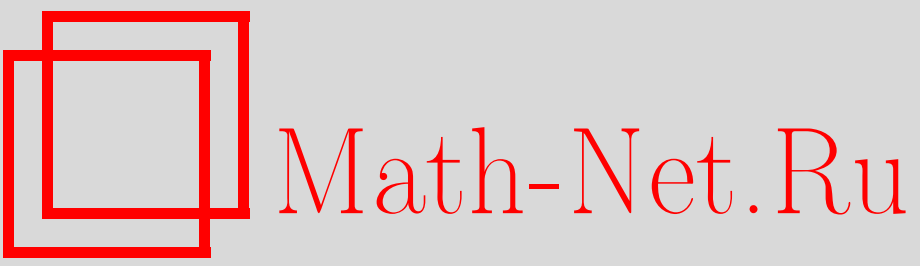

Н. Шпак, Удержание решений нелинейных волновых уравнений промежуточными аттракторами, ТМФ, 2001, том 127, номер 3, 475-487

DOI: https://doi.org/10.4213/tmf475

Использование Общероссийского математического портала Math-Net.Ru подразумевает, что вы прочитали и согласны с пользовательским соглашением

http://www . mathnet.ru/rus/agreement

Параметры загрузки:

IP : 34.239 .49 .27

26 апреля 2023 г., 15:22:02 
ТЕОРЕТИЧЕСКАЯ

И МАТЕМАТИЧЕСКАЯ

ФИЗИКА

Том 127, № 3

июнь, 2001

(C) 2001 г.

Н. Шпак*

\section{УДЕРЖАНИЕ РЕШЕНИЙ НЕЛИНЕЙНЫХ ВОЛНОВЫХ УРАВНЕНИЙ ПРОМЕЖУТОЧНЫМИ АТТРАКТОРАМИ}

Исследуется динамика нелинейных волновых уравнений вблизи порога образования сингулярности. Интерес к таким исследованиям вызван неожиданными свойствами, наблюдаемыми в критических явлениях, связанных с гравитационным коллапсом. Детально исследуется одно из таких свойств, а именно возникновение в динамике универсального промежуточного аттрактора вблизи порога формирования сингулярности. Получившаяся эволюционная картина имеет следующий вид: при всех начальных данных, достаточно близких к порогу образования сингулярности, система стремится к универсальному статическому решению, играюшему роль промежуточного аттрактора. В течение конечного времени удержания, зависящего явным образом от начальных данных, система остается вблизи этого решения, а затем продолжает эволюцию и в конечном итоге либо образует сингулярность, либо распадается (диспергирует). Удержание, равно как и уход из области аттрактора, описывается в линейном приближении квазинормальными модами (KHM) и одной растущей модой. Более того, вычисленные в линейном приближении (с помощью линейных обыкновенных дифференциальных уравнений) профили мод, возникающих в порядках теории возмущений, частоты осцилляций этих мод и факторы, подавляющие потенциально растущие множители, согласуются с численными расчетами эволюционных процессов, описываемых нелинейными дифференциальными уравнениями в частных производных. В частности, показатель экспоненциального распада растущей моды задает время существования промежуточного состояния (вблизи аттрактора), в то время как несколько наименее подавленных КНМ проявляют себя в релаксационных процессах как возмущения.

\section{1. ВВЕДЕНИЕ}

В обшей теории относительности эволюция изолированной системы, такой как звезда или ограниченное в пространстве распределение какого-либо типа материи (задаваемое своим тензором напряжений), или даже просто гравитационное поле, как правило, приводит в итоге к стационарному (конечному) состоянию: система или коллапсирует, или полностью распадается, или, возможно, образует звезду (под которой понимается любое локализованное стабильное распределение материи). Образование звезды, т.е.

*Institut für Theoretische Physik, J.W. Goethe Universität, Frankfurt am Main, Germany. E-mail: szpak@th.physik.uni-frankfurt.de 
регулярного решения с пустым пространством вокруг него, в дальнейшем рассмотрении не будет отличаться от случая полного распада с образованием пустого пространства. В эвристической картине эволюции система с “большими” начальными данными в итоге преврашается в черную дыру (за конечное собственное время образуется сингулярность), в то время как система с "малыми" начальными данными распадается посредством излучения, оставляя пространство пустым и плоским в бесконечно удаленном будушем (или образуя решение типа звезды). Таким образом, фазовое пространство всех решений разбивается на области притяжений: почти любой набор начальных данных приводит в итоге к одному из стабильных конечных состояний. Возникает вопрос о том, что происходит в промежуточном случае, т.е. на границе между областью образования черной дыры и областью дисперсии в пустое пространство. Интересным и, по-видимому, наиболее простым примером служит процесс сферически-симметричного гравитационного коллапса вешественного скалярного поля. В зависимости от начальных данных (плотности энергии скалярного поля) этот процесс либо приводит к образованию шварцшильдовской черной дыры, либо к бесконечно распределенному полю в пустом и плоском пространстве. В своей основополагаюшей работе [1] Чоптюк исследовал окрестность границы между областями, отвечающими этим двум ветвям эволюции. Он обнаружил, что решение на пороге области коллапса оказывается универсальным для всех критических начальных данных, т.е. что для всех возможных критических начальных данных, попадаюших точно в граничную область, решение эволюционирует к одному и тому же решению. Это решение инвариантно относительно дискретных преобразований автодуальности для некоторого универсального масштабного параметра. Такие же или очень похожие схемы критической динамики были впоследствии найдены во многих других моделях, в которых материя взаимодействует с гравитацией таким образом, что возможны варианты эволюции, приводящие либо к образованию черной дыры, либо к полной дисперсии (по поводу последних достижений в этой области см. обзор [2])

Схема эволюции была доказана аналитически в некоторых простых случаях (например, для сферически-симметричного гравитируюшего безмассового скалярного поля [3]), но в более общих случаях известны лишь результаты численных выгислений. Уравнения эволюции как гравитационного поля, так и полей материи оказываются слишком сложными и не поддаются аналитическим расчетам (по крайней мере, с помошью современных точных методов). По этой причине целый ряд упрошенных моделей исследовался в надежде достигнуть лучшего понимания явлений, происходящих в гравитирующих системах. Основной вопрос состоит в том, какие свойства критических решений гравитируюших систем оказываются присушими нелинейным эволюционным уравнениям, а какие связаны непосредственно с гравитацией (уравнениями Эйнштейна). В упрошенных моделях решения типа черных дыр отвечают образованию сингулярности, т.е. бесконечного роста полей или их производных за конечное время. До настояшего времени не построено ни одной модели, допускающей дискретное автодуальное решение в граничной области между областями образования черной дыры и дисперсии. Таким образом, основная проблема, исследуемая в данной статье - это проблема универсаль- 
ности: является ли сушествование универсального критического решения, играюшего роль промежуточного аттрактора в критической динамике, обшим свойством, присушим нелинейным эволюционным уравнениям?

Оказалось, что упрошенные модели, которые исследуются в данной работе, обладают следующим общим свойством: если эволюция начинается из области вблизи порога образования черной дыры, то система сначала эволюционирует в универсальное промежуточное состояние и лишь потом, по прошествии конечного времени, либо диспергирует, либо образует сингулярность. Промежуточное состояние - это решение с единственной растущей пертурбативной модой (такое решение называется критическим). $\mathrm{C}$ точки зрения теории динамических систем критическое решение - это аттрактор для систем, лежащих в точности на пороге образования сингулярности, который является гиперповерхностью коразмерности 1 в полном фазовом пространстве решений. Этот аттрактор оказывает отталкивающее действие (в обе стороны) в направлениях, перпендикулярных этой гиперповерхности.

Ранее исследовались [4] несколько типов нелинейных волновых уравнений вида

$$
\square u+f(u)=0,
$$

где $u$ - вещественное скалярное поле, а $f$ - нелинейная непрерывная функция такая, что $f(0)=0$.

Некоторые аналогичные схемы эволюции, такие как образование сингулярности в случае "больших" начальных данных и глобальное сушествование решений в случае "малых" начальных данных (см. обзор [5]), были доказаны для различных типов нелинейности.

\section{2. ИССЛЕДУЕМЫЕ УРАВНЕНИЯ}

В данной работе анализируются следующие модели:

$$
\begin{aligned}
\square u-u^{5} & =0, \\
\square u+u-u^{3} & =0, \\
\square u+u-u^{3}+B u^{5} & =0,
\end{aligned}
$$

и рассмотрение для простоты ограничивается сферически-симметричным случаем. Во всех этих моделях $f(-u)=-f(u)$, что обеспечивает глобальную симметрию $u \rightarrow-u$, выполненную для всех решений. Тем самым без потери общности можно исследовать только одно решение из пары, поскольку решения из пары оказываются симметричными друг другу.

Уравнение (NW) - это нелинейное волновое уравнение, в то время как уравнения $(\mathrm{NKG})$ и $(\mathrm{NKG} B)$ являются скорее нелинейными уравнениями Клейна-Гордона ввиду наличия линейного члена в функции $f(u)$. Нет никакой необходимости удерживать в уравнениях какие-либо константы, поскольку все константы, кроме постоянной $B$ в уравнении (NKG $B)$, варьируя которую можно качественно менять решения, как оказывается, можно сделать единичными с помошью растяжек координат и полей. 
Численные вычисления эволюции решения, начальные данные которого имеют компактный носитель, простираюшийся до начала координат $(r=0)$, привели к гипотезе, что сушествуют два конечных состояния: $u(t, r)=0$ (дисперсия) и $u(t, r=0) \rightarrow \infty$ (сингулярность в начале координат). В модели (NKG $B$ ) конечные состояния имеют вид

$$
u(t, r)=0, \quad u(t, r)=\sqrt{\frac{1+\sqrt{1-4 B}}{2 B}} .
$$

Ни одно из этих состояний не сингулярно, но эти состояния хорошо разделены (не сушествует непрерывного семейства стационарных решений, связываюшего эти два состояния) и стабильны. Поэтому каждое из этих решений имеет свою собственную область притяжения в фазовом пространстве, и пороговая область между этими областями может быть исследована аналогично.

Явньй вид “потенциальной” энергии

$$
\int d^{3} x F(u)
$$

в функционале энергии

$$
E=\int d^{3} x\left[\frac{1}{2} \dot{u}^{2}+\frac{1}{2}(\nabla u)^{2}+F(u)\right]
$$

где $F^{\prime}(u)=f(u)$, предполагает, что неограниченный рост величины поля возможен в моделях (NW) и (NKG), но невозможен в модели (NKGB). Причина этого заключена в знаке $F(u)$ при $|u| \rightarrow \infty$, поскольку именно этот знак определяет, ограничен ли функционал энергии снизу. В случае уравнения (NKGB) функционал энергии ограничен снизу, это приводит к тому, что первая соболевская норма ограничена (если только конечна энергия начального состояния). В этом случае решение остается регулярным и конечным при всех временах.

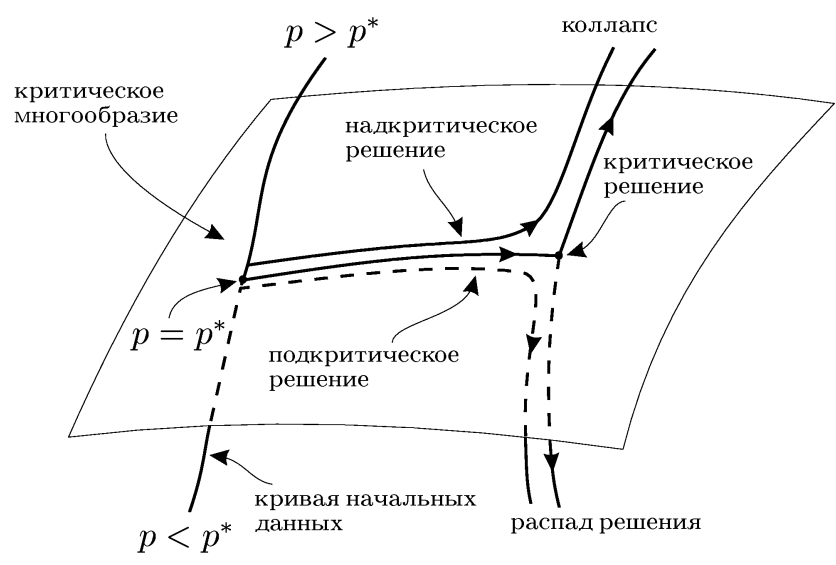

Рис. 1. Стабильное подмногообразие критических начальных данных коразмерности 1. 
Рассматривая эволюцию однопараметрического семейства решений с начальными данными (простираюшимися до начала координат и имеюшими компактный носитель и конечную энергию) такого, что одно из конечных состояний эволюции (сингулярность) достигается при некоторых значениях параметра $p$, а другое конечное состояние (пустое пространство) достигается при других значениях $p$, можно с помощью бисекций найти критическое значение $p^{*}$, для которого критические начальные данные, равно как и траектория эволюции решения, содержатся внутри границы между двумя областями притяжения (рис. 1). Например, в качестве начального распределения можно выбрать распределение Гаусса с амплитудой $p$. Тогда при малых значениях $p \approx 0$ поле в итоге диспергирует, а при больших $p$ оно достигает другого стабильного состояния (коллапсирует или переходит к какому-либо статическому состоянию, зависящему от рассматриваемой модели). Выбирая различные семейства начальных данных (различные формы гауссианы или других функций) и определяя критическое значение для каждого такого семейства, можно найти и исследовать границу в фазовом пространстве между областями притяжения двух стабильных конечных состояний.

Рассмотрим более детально уравнение (NW), потому что многие результаты в этом случае могут быть получены аналитически. Затем обсудим отличия, возникающие в случае двух других уравнений, в частности, из-за наличия линейного члена в функции $f(u)$.

\section{3. ПРОМЕЖУТОЧНЫЙ АТТРАКТОР}

Численные расчеты показали, что решение с малой начальной амплитудой отражается от начала координат $r=0$ и затем излучается в бесконечность, так что конечное состояние тривиально: $u(t, r)=0$. При больших начальных амплитудах происходит коллапс в начале координат: $u(t, r=0) \rightarrow \pm \infty$. Поиск начальных данных с критической амплитудой на пороге коллапса всегда приводит к статическому решению, играюшему роль промежуточного аттрактора.

Аналитические вычисления дают всего лишь одно статическое решение (с точностью до масштабных преобразований $\left.{ }^{1)}\right)$ :

$$
u_{a}(r)=\frac{a}{\sqrt{1+a^{4} r^{2} / 3}} .
$$

Эти статические решения, изображенные на рис. 2 , наблюдались также и в численных динамических расчетах. Однако предсказать аналитически, исходя из начальных данных, какое именно статическое решение из однопараметрического семейства (задаваемого параметром $a$ ) получается в результате эволюции, оказалось невозможно. Тем не менее возможны некоторые оценки, поскольку решения с различными масштабными параметрами отвечают различным величинам некоторой сохраняющейся величины, отвечающей нётеровскому току для соответствующих масштабных преобразований, -

\footnotetext{
1) Заметим, что в уравнении (NW) имеется смешанная масштабная симметрия: $u(t, r) \rightarrow$ $a u\left(a^{2} t, a^{2} r\right)$.
} 


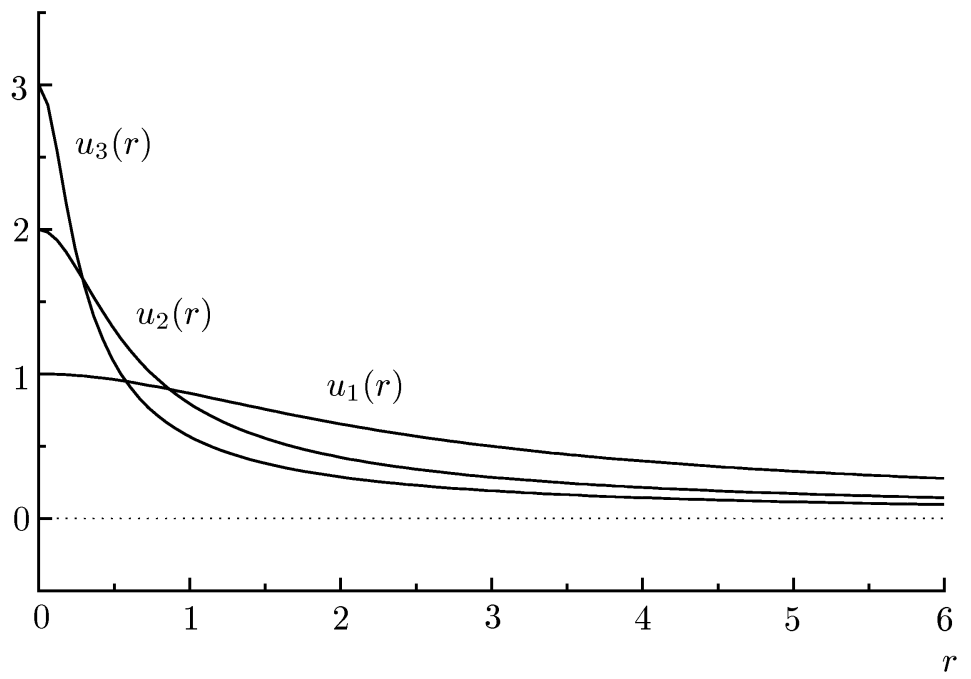

Рис. 2. Статические решения с различными масштабными параметрами.

симметрии соответствующего уравнения. Эта величина может быть легко вычислена исходя из начальных данных; проблема в том, что она не остается постоянной в рассматриваемой окрестности начала координат, поскольку она частично излучается в бесконечность вместе с той частью поля, которая теряется в виде излучения до достижения системой точки аттрактора.

Как принято в случае промежуточного аттрактора, возмушенные статические решения имеют ровно одну растушую моду. Чтобы это показать, рассмотрим линейное приближение динамики поля $u(t, r)$ вблизи статического решения $u_{a}(r)$ и введем возмушение:

$$
u(t, r)=u_{a}(r)+\frac{\phi(t, r)}{r} .
$$

После подстановки в уравнение (NW), удерживая только линейные по $\phi$ члены, получим линейное волновое уравнение

$$
\phi_{t t}-\phi_{r r}+V_{a}(r) \phi=0
$$

в котором величина

$$
V_{a}(r)=f^{\prime}\left(u_{a}(r)\right)=-5\left[u_{a}(r)\right]^{4}=\frac{-5 a^{4}}{\left(1+\frac{a^{4} r^{2}}{3}\right)^{2}}
$$

играет роль эффективного потенциала. Производя преобразование Фурье по переменной времени (или выделяя временну́ю зависимость $\phi(t, r)=e^{-i k t} \psi(r)$ ), придем к задаче на собственные значения

$$
-\psi_{r r}+V_{a}(r) \psi=k_{a}^{2} \psi
$$




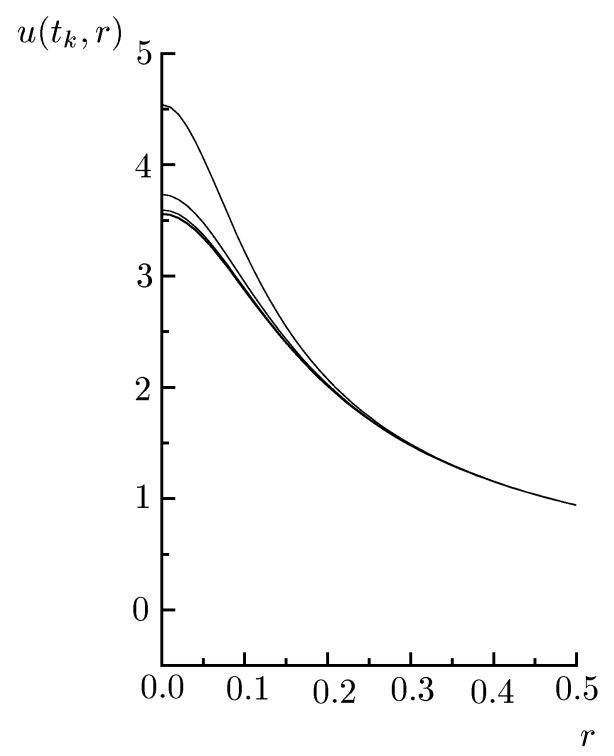

Рис. 3. Уход решения из области промежуточного аттрактора: изображено статическое решение с добавленной растущей модой (с положительной амплитудой) для последовательности равноудаленных моментов времени $t_{k}$. "Время жизни" и скорость удаления описываются ляпуновской экспонентой $\lambda_{a}=a^{2} 1.100167$.

У этого уравнения есть аналитическое решение при $k_{a}=0$ :

$$
\psi_{a}(r)=r\left(1-\frac{a^{4}}{3} r^{2}\right)\left(1+\frac{a^{4}}{3} r^{2}\right)^{-\frac{3}{2}},
$$

и это решение обрашается в нуль при $r=\sqrt{3} / a^{2}$. Тогда из теории Штурма-Лиувилля следует, что у потенциала $V_{a}(r)$ имеется ровно одно "связанное состояние", т.е. ограниченное решение $\psi(r)$, убываюшее на бесконечности с $k_{a}^{2}<0$. Тогда $k_{a}= \pm i \lambda_{a}$ с вешественной константой $\lambda_{a}$. С помошью масштабных преобразований получим, что $\lambda_{a}=a^{2} \lambda_{1}$. Численный расчет дает значение $\lambda_{1}=1.100167$. Возврашаясь к переменным времени (с помощью обратного преобразования Фурье), увидим, что один из двух возможных выборов знака $k$ порождает экспоненциально растущую моду:

$$
\phi(t, r)=e^{\lambda t} \psi(r)
$$

где $\lambda$ - показатель Ляпунова растущей моды теории возмущений, задающий время жизни решения вблизи промежуточного аттрактора. Численные расчеты эволюции решений подтвердили как экспоненциальный характер распада промежуточного состояния, так и численное значение показателя экспоненты $\lambda$. При этом явно наблюдалась и сама функция $\psi(r)$ в виде растушего возмушения (рис. 3$)$.

\section{4. ДИНАМИКА ВБЛИЗИ АТТРАКТОРА}

Ожидается, что вблизи аттрактора линейное приближение задает правильное описание динамики. Сравнение с численными расчетами показывает, что в действитель- 
ности это приближение оказывается на удивление точным. Решение достигает промежуточного аттрактора очень быстро и оказывается в достаточной близости от статического решения, для того чтобы линейное приближение давало ведуший вклад.

Линеаризованное уравнение имеет вид (2). Процессы релаксации, обусловленные излучением на бесконечность, могут быть описаны квазинормальными модами (КНМ). Эти моды суть решения задачи на собственные значения (3), которые удовлетворяют динамическому условию для волн, уходяших на бесконечность, т.е. пропорциональны $e^{i k r}$ при $r \rightarrow \infty$. Эта задача не является задачей Штурма-Лиувилля, а потому собственные значения $k^{2}$ не обязательно вешественны. В обшем случае эти параметры оказываются комплексными числами: $k=\omega-i \Gamma$, где $\omega$ и $\Gamma$ - вешественные параметры, $\Gamma>0$. Поэтому пространственная асимптотика КНM на бесконечности имеет вид $e^{i \omega r} e^{\Gamma r}$, и эти моды оказываются неограниченными, что порождает серьезные трудности при вычислениях и даже при определении КНМ как решений с чисто экспоненциально растушей асимптотикой, поскольку остальные линейно независимые решения экспоненциально убывают и тем самым оказываются малыми по сравнению с КНM. Корректное математическое описание КНМ было дано Ноллертом и Шмидтом [6] с помощью преобразования Лапласа

$$
\hat{\phi}(s, r)=\int_{0}^{\infty} e^{-s t} \phi(t, r) d t
$$

и полюсов функции Грина. Преобразованное уравнение имеет вид

$$
s^{2} \hat{\phi}-\hat{\phi}_{r r}+V \hat{\phi}=s g(r)+h(r) \equiv j(s, r),
$$

где правая часть представляет начальные данные $g(r)=\phi(0, r)$ и $h(r)=\phi_{t}(0, r)$. Неоднородное линейное уравнение (4) может быть решено с использованием аппарата функций Грина. Подходящая функция Грина, удовлетворяюшая граничным условиям, может быть построена из двух линейно независимых решений однородной части уравнения (4), каждое из которых удовлетворяет одному граничному условию. Обозначим эти решения через $\hat{\phi}_{1}(r)$ и $\hat{\phi}_{2}(r)$ и наложим условия регулярности $\hat{\phi}_{1}(0)=0$ и $\hat{\phi}_{2}(r) \sim e^{-s r}$ при $r \rightarrow \infty$. Тогда функция Грина может быть определена следующим образом:

$$
\widehat{G}\left(s, r, r^{\prime}\right)=\frac{1}{W\left[\hat{\phi}_{1}, \hat{\phi}_{2}\right]} \cdot \begin{cases}\hat{\phi}_{1}(s, r) \hat{\phi}_{2}\left(s, r^{\prime}\right), & r<r^{\prime}, \\ \hat{\phi}_{1}\left(s, r^{\prime}\right) \hat{\phi}_{2}(s, r), & r>r^{\prime},\end{cases}
$$

и обшее решение выражается через начальные данные стандартным образом:

$$
\hat{\phi}(s, r)=\int \widehat{G}\left(s, r, r^{\prime}\right) j\left(r^{\prime}\right) d r^{\prime} .
$$

Возмущенная часть получается после обратного преобразования Лапласа

$$
\begin{aligned}
\phi(t, r) & =\mathcal{L}^{-1}[\hat{\phi}(s, r)]=\mathcal{L}^{-1}\left[\int d r^{\prime} \widehat{G}\left(s, r, r^{\prime}\right) j\left(r^{\prime}\right)\right]= \\
& =\int d r^{\prime}\left\{f\left(r^{\prime}\right) \mathcal{L}^{-1}\left[s \widehat{G}\left(s, r, r^{\prime}\right)\right]+g\left(r^{\prime}\right) \mathcal{L}^{-1}\left[\widehat{G}\left(s, r, r^{\prime}\right)\right]\right\}= \\
& =\int d r^{\prime}\left[f\left(r^{\prime}\right) \partial_{t} G\left(t, r, r^{\prime}\right)+g\left(r^{\prime}\right) G\left(t, r, r^{\prime}\right)\right]
\end{aligned}
$$


в котором функция

$$
G\left(t, r, r^{\prime}\right)=\mathcal{L}^{-1}\left[\widehat{G}\left(s, r, r^{\prime}\right)\right]=\int_{C} e^{s t} \widehat{G}\left(s, r, r^{\prime}\right) d s
$$

содержит всю ту информацию о динамике, которая не зависит от начальных данных. Контур интегрирования в этой формуле параллелен мнимой оси и обходит все полюсы справа. Можно замкнуть контур интегрирования в левой половине комплексной плоскости, после чего результатом интегрирования будет сумма вычетов во всех полюсах $\widehat{G}$, если только функция Грина не имеет разрезов, что имеет место в указанном случае. Ответ имеет вид

$$
G\left(t, r, r^{\prime}\right)=a e^{\lambda t} \chi\left(r, r^{\prime}\right)+\sum_{k} b_{k} e^{-\Gamma_{k} t} e^{i \omega_{k} t} \phi_{k}\left(r, r^{\prime}\right)
$$

где первый член происходит из полюса в правой части комплексной плоскости $\operatorname{Re} s=$ $\lambda>0$ и представляет собой растушую моду. Второй член происходит из суммы по всем полюсам с Re $s<0$ и представляет собой вклад всех убывающих КНM. Коэффициенты $a$ и $b_{k}$ суть вычеты в соответствуюших полюсах, а функции $\chi(r)$ и $\phi_{k}(r)$ - это соответствуюшие профили растушей моды и КНМ.

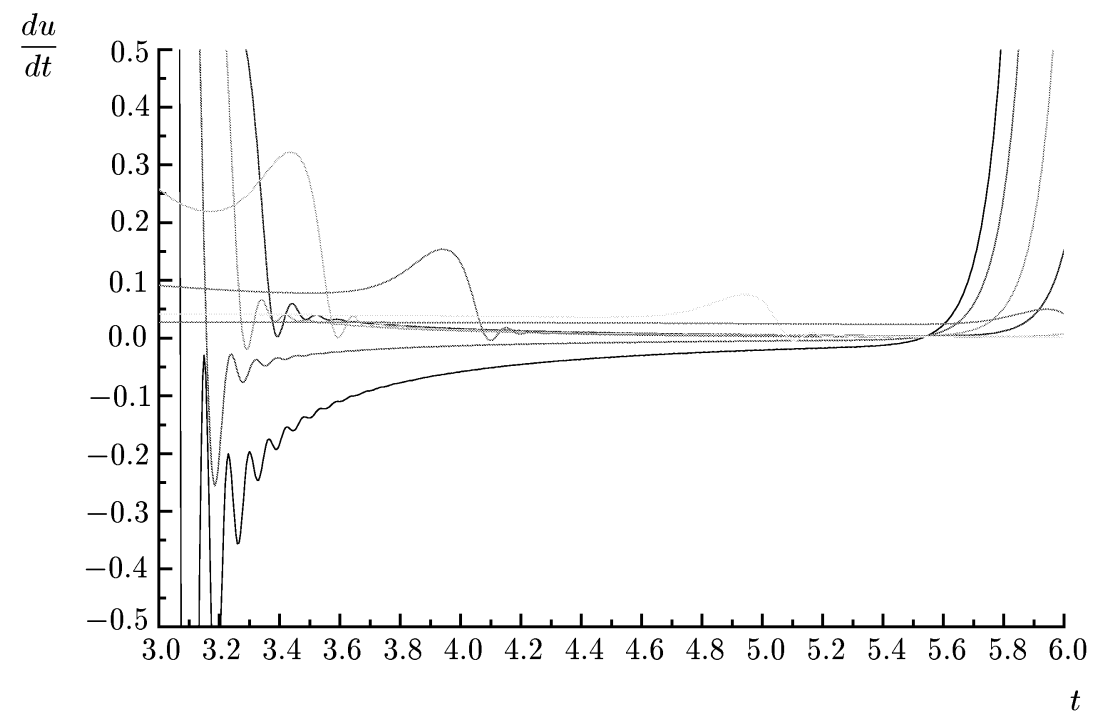

Рис.4. Динамика возмушений в выделенных точках. На двух наиболее низко расположенных кривых (отвечающих точкам, наиболее приближающимся к началу координат) отчетливо видны две основные КНМ, первая из которых чисто убывающая, а вторая убывает и одновременно осциллирует. В дальнейшем развивается возрастающая мода.

Окончательный ответ для эволюции решения вблизи аттрактора имеет вид

$$
u(t, r)=u_{a}(r)+A(p) e^{\lambda t} \chi(r)+\sum_{k} B_{k}(p) e^{-\Gamma_{k} t} e^{i \omega_{k} t} \phi_{k}(r)
$$


где функции $A(p)$ и $B_{k}(p)$ теперь уже зависят от начальных данных и, в частности, от параметра $p$, задаюшего однопараметрическое семейство начальных данных. С помощью тонкой подгонки параметра $p \rightarrow p^{*}$ можно добиться того, что $A(p) \approx 0$ (в то время как $\left.A\left(p^{*}\right)=0\right)$. В этом случае растущая мода развивается достаточно медленно и можно наблюдать динамику поведения решения вблизи аттрактора. В реальных вычислениях удается наблюдать несколько наименее подавленных КНМ. Именно эти моды доминируют на поздних этапах приближения решения к промежуточному аттрактору до тех пор, пока не развилась растущая мода.

Ведущая (наименее подавленная) КНМ оказывается не осциллируюшей; численная оценка дала для показателя ее убываюшей экспоненты оценку $\Gamma_{1}=0.078$. Этот параметр меняется при масштабных преобразованиях так же, как и $\lambda_{a}$, т.е. $\Gamma_{a}=a^{2} \Gamma_{1}$. На рис. 4 изображен случай $a=3.594$, что отвечает $\Gamma_{a}=1.014$.

"Время жизни" решения вблизи промежуточного аттрактора определяется показателем экспоненты $\lambda_{a}$ нестабильной моды и расстоянием от аттрактора в фазовом пространстве, в терминах начальных данных оно имеет вид

$$
T \approx-\frac{1}{\lambda_{a}} \ln \left|p-p^{*}\right|
$$

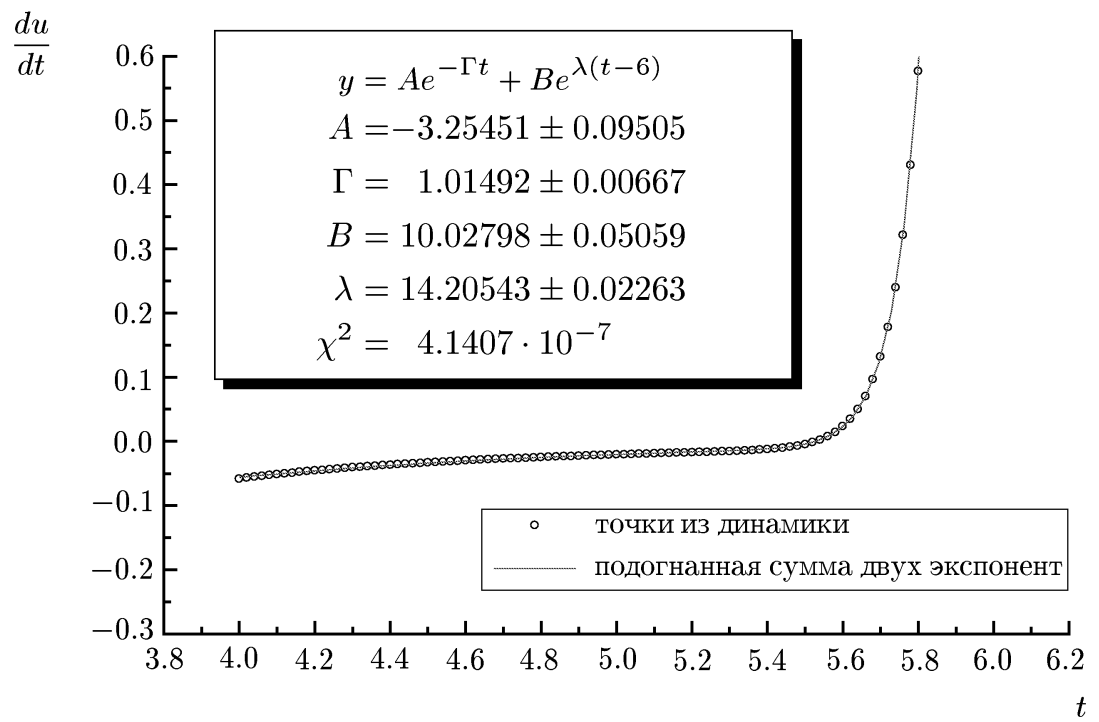

Рис. 5. Длительное пребывание решения вблизи аттрактора, обеспеченное с помощью неосциллирующей подавленной KHM, в итоге прерывается с развитием растущей моды. Значения подогнанных показателей экспонент $\lambda$ и $\Gamma$ находятся в согласии с результатами других численных расчетов.

\section{5. НЕЛИНЕЙНОЕ УРАВНЕНИЕ КЛЕЙНА-ГОР ДОНА}

Нелинейное уравнение Клейна-Гордона отличается от нелинейного волнового уравнения наличием линейного члена, например $m^{2} u$, в функции $f(u)$ в уравнении (1). Нели- 


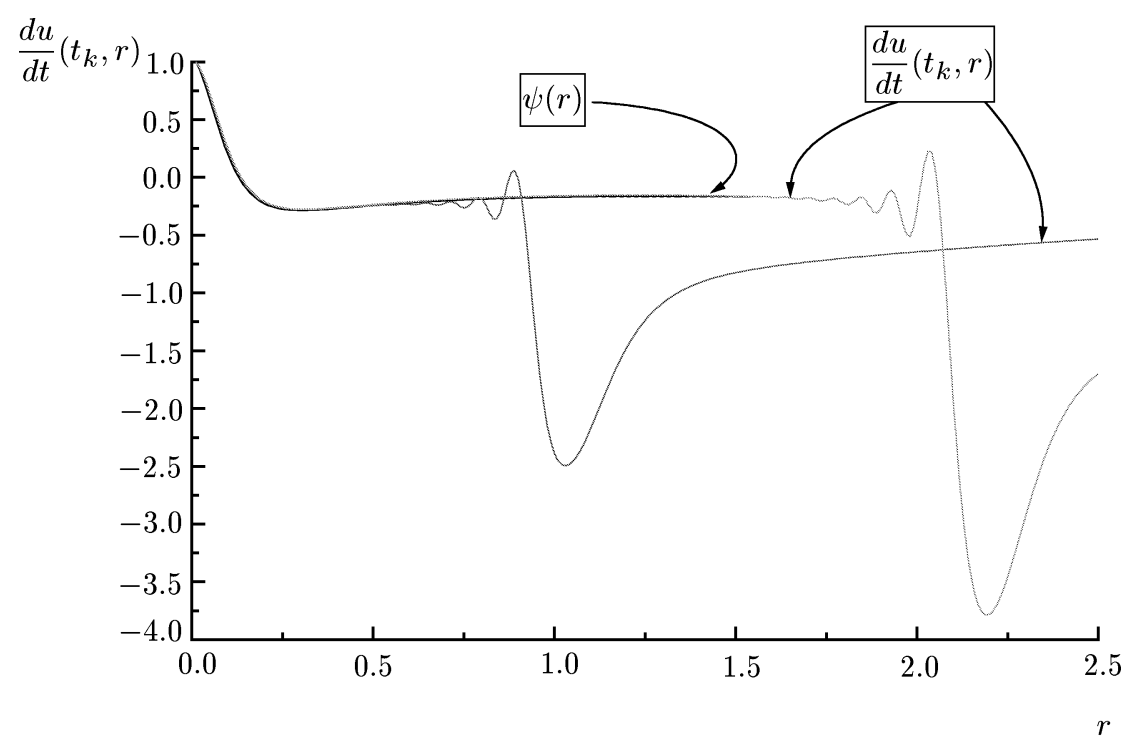

Рис.6. Профиль функции основной КНМ $\psi(r)$ в сравнении с мгновенными профилями динамического решения, полученными при двух выделенных временах $t_{k}$. С течением времени функция динамического решения приближается к уровню вычисленной КНM, начиная с точки начала отсчета. Этот процесс прерывается резким (экспоненциальным) уходом решения из области промежуточного аттрактора; поэтому хвосты функций профиля не играют роли в настоящей динамике.

нейная схема эволюции при этом в основном сохраняется, но процесс приближения и релаксации решения вблизи промежуточного аттрактора при этом меняется, поскольку в указанном случае решения не содержат КНМ. Причина этого в том, что у функции Грина появляется разрез между точками $+i m$ и $-i m$ в комплексной плоскости. Вклад этого разреза в интеграл в обратном преобразовании Лапласа описывается дополнительным членом, пропорциональным $\theta\left(t^{2}-r^{2}\right) J_{0}\left(m \sqrt{t^{2}-r^{2}}\right)$. Этот член имеет асимптотическое поведение, пропорциональное $\cos (m t) t^{-3 / 2}$ при больших временах и, следовательно, убывает медленнее любой экспоненты и подавляет все КНМ. При этом в решении наблюдаются только возмущения, осциллирующие с частотой $m$ и убывающие степенным образом; как и раньше, этот процесс релаксации прерывается с развитием растущей моды, после чего решение покидает область промежуточного аттрактора (рис. 5, 6).

\section{6. ЗАМЕЧАНИЯ}

С точки зрения наблюдателя, достаточно удаленного от начала координат, динамика поля вблизи аттрактора имеет следующий вид: сначала поступает сигнал непосредственно из точки начальных данных, а потом поступающие сигналы приобретают универсальный характер. Они состоят из экспоненциально убывающих квазинормальных осцилляций и из растушей моды. Начальные данные дают вклад только в амплитуды отдельных мод. Тогда из информации о частотах КНМ в случае черных дыр в гравитируюших системах можно извлечь все параметры черной дыры, такие как массу, заряд 

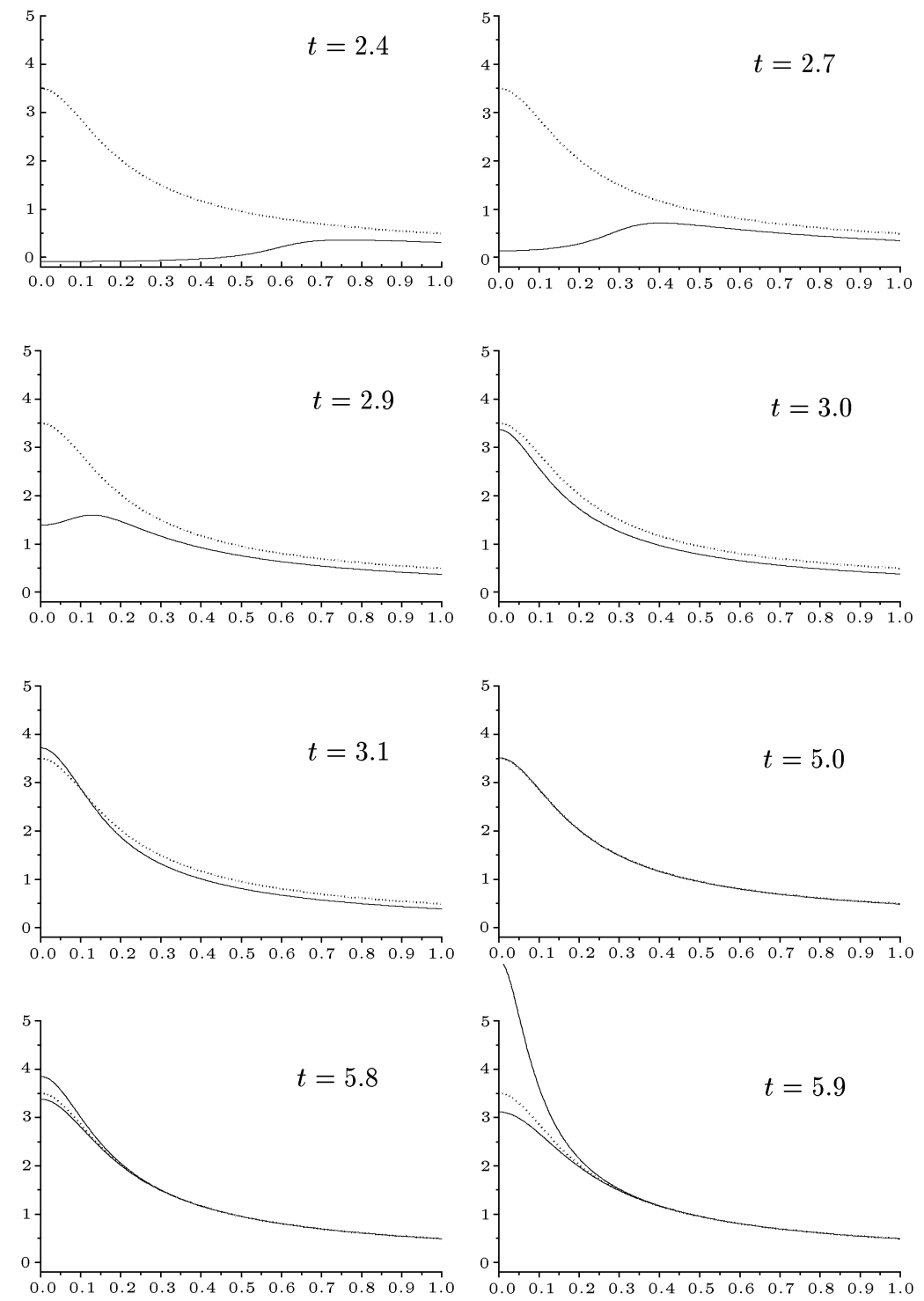

Рис. 7. Эволюция до- и надкритических начальных данных, которые изначально близки друг к другу, но в конечном итоге приводят к различным конечным состояниям. Промежуточный аттрактор изображен пунктирной линией.

и угловой момент. В случае уравнения $(\mathrm{NW})$ наблюдается схожее явление: существует однопараметрическое семейство промежуточных аттракторов, которые суть одно и то же статическое решение, подвергнутое некоторому масштабному преобразованию, и известна аналитическая зависимость частот КНМ от этого масштабного преобразования. Наблюдая сигнал и определяя частоты и параметры затухания осцилляций, можно определить, к какому именно аттрактору стремится система (рис. 7). 


\section{7. ЗАКЛЮЧЕНИЕ}

Показано, что нелинейные волновые уравнения (или уравнения Клейна-Гордона) в случае сушествования по крайней мере двух различных областей притяжения (аттракторов) в картине глобальной эволюции и, следовательно, граничного подмногообразия коразмерности 1 в фазовом пространстве имеют в точности одно статическое решение на этом подмногообразии. В критической динамике это решение играет роль универсального промежуточного аттрактора. Отсюда можно заключить, что универсальность динамики в критическом гравитационном коллапсе не является свойством, присущим только уравнениям Эйнштейна, а напротив, является общим свойством для целого класса достаточно простых нелинейных волновых уравнений.

Другие свойства критического гравитационного коллапса, такие как дискретная автоморфность решений, остаются нерешенными проблемами.

Благодарности. Автор благодарит профессора Петра Бизоня (Институт физики Ягеллонского университета, Краков) за постановку задачи, постоянную поддержку и многочисленные обсуждения в процессе работы над обсуждаемой проблемой.

\section{Список литературы}

[1] M. W. Choptuik. Phys. Rev. Lett. 1993. V. 70. P. 9

[2] C. Gundlach. Critical phenomena in gravitational collapse: Living reviews. gr-qc/0001046.

[3] D. Christodoulou. Commun. Pure Appl. Math. 1991. V. 44. P. 339; 1993. V. 46. P. 1131.

[4] N. Szpak. Relaxation to unstable attractors in nonlinear wave equations. Master's thesis. Cracow: Jagellonian University, 1999.

[5] W. A. Strauss. Nonlinear Wave Equations. Conference Board of the Mathematical Sciences. V. 73. Providence, RI: AMS, 1989.

[6] H.-P. Nollert, B. G. Schmidt. Phys. Rev. D. 1992. V. 45. P. 2617. 